\title{
Choosing the probe radiation wavelengths for noninvasive monitoring of haemoglobin in blood
}

\author{
Igor L. Davydkin ${ }^{1}$, Natalia S. Kozlova ${ }^{1 *}$, Vadim N. Koniukhov ${ }^{2}$, Tatiana P. Kuzmina ${ }^{1}$, \\ and Elizaveta V. Mordvinova ${ }^{1}$ \\ ${ }^{1}$ Samara State Medical University, 89 Chapaevskaya str., Samara 443099, Russia \\ ${ }^{2}$ Samara State Aerospace University, 34 Moskovskoe shosse, Samara 443086, Russia \\ *e-mail: gorachaia@live.ru
}

\begin{abstract}
The goal of our study was to estimate the accuracy of measuring the blood haemoglobin concentration by means of the pulse CO-oximetry, depending on the probe radiation wavelength.

Revealing the combinations of wavelengths, optimal for minimising the measurement error, will allow the formulation of requirements to the instruments for noninvasive assessment of haemoglobin concentration, applicable in wide clinical practice. To solve this problem, we examined a group of haematological patients in the Haematology Department of the Samara State Medical University Clinic with the aim to compare the results obtained using the experimental multiwave system for noninvasive haemoglobin concentration assessment and the results of the clinical blood analysis. (C) 2016 Journal of Biomedical Photonics \& Engineering.
\end{abstract}

Keywords: monitoring, hemoglobin, noninvasive diagnosis, laser.

Paper \#2835 received 2015.12.23; revised manuscript received 2016.03.10; accepted for publication 2016.03.10; published online 2016.03.31. doi: 10.18287/JBPE16.02.010305

\section{References}

1. J. W. McMurdy, G. D. Jay, S. Suner, and G. Crawford, "Noninvasive Optical, Electrical, and Acoustic Methods of Total Hemoglobin Determination," Clinical Chemistry 54(2), 264-272 (2008).

2. K. Saigo, S. Imoto, M. Hashimoto, H. Mito, J. Moriya, T. Chinzei, Y. Kubota, S. Numada, T. Ozawa, and S. Kumagai, "Noninvasive Monitoring of Hemoglobin: The Effects of WBC Counts on Measurement," Am. J. Clin. Pathol. 121(1), 51-55 (2004).

3. E. V. Kozhokina, "Noninvasive methods of measuring bilirubin, haemoglobin, and glucose. The haemobiliglucometer instrument," Scientific and Technical Journal of Information Technologies, Mechanics and Optics 2(72), 157-162 (2011). [in Russian].

4. R. Kumar, and H. Ranganathan, "Noninvasive Sensor Technology for Total Hemoglobin Measurement in Blood," Journal of Industrial and Intelligent Information 1(4), 243-246 (2013).

5. R. Doshi, and A. Panditrao, "Non-Invasive Optical Sensor for Hemoglobin Determination," International Journal of Engineering Research and Applications 3(2), 559-562 (2013).

6. J. E. Bender, A. B. Shang, E. W. Moretti, B. Yu, L. M. Richards, and N. Ramanujam, "Noninvasive monitoring of tissue hemoglobinusing UV-VIS diffuse reflectance spectroscopy: a pilot study," Optics Express 17(26), 23396-23409 (2009).

7. U. Timm, G. Leen, E. Lewis, D. McGrath, J. Kraitl, and H. Ewald, "Non-Invasive Optical Real-time Measurement of Total Hemoglobin Content," Procedia Engineering 5, 488-491 (2010).

8. D. J. Deyo, R. O. Esenaliev, O. Hartrumpf, M. Motamedi, and D. S. Prough, "Continuous noninvasive optoacoustic monitoring of hemoglobin concentration," Anesthesiol Analgesia 92(S139), (2001).

9. I. Y. Petrova, R. O. Esenaliev, Y. Y. Petrov, H. P. E. Brecht, C. H. Svensen, J. Olsson, D. J. Deyo, and D. S. Prough, "Optoacoustic monitoring of blood hemoglobin concentration: a pilot clinical study," Opt. Lett. 30(13), 1677-1679 (2005).

10. G. Lindner, and A. K. Exadaktylos, "How Noninvasive Haemoglobin Measurement with Pulse CO-Oximetry Can Change Your Practice: An Expert Review,” Emerg. Med. Int. 2013, 701529 (2013). 
11. D. Frasca, C. Dahyot-Fizelier, K. Catherine, Q. Levrat, B. Debaene, and O. Mimoz, "Accuracy of a Continuous Noninvasive Hemoglobin Monitor in Intensive Care Unit Patients," Crit. Care Med. 39(10), 2277 2282 (2011).

12. Rad-57. Signal Extraction Pulse CO-Oximeter, Operator's manual (2011).

13. Radical-7. Signal Extraction Pulse CO-Oximeter, Operator's manual (2010).

14. S. Prahl, "Optical absorption of hemoglobin," Oregon Medical Laser Center (1999).

15. O. Siggaard-Andersen, B. Norgaard-Pedersen, and J. Rem, "Hemoglobin pigments spectrophotometric determination of oxy-, carboxy-, met-, and sulfhemoglobin in capillary blood," Clinica Chimica Acta 42(1), 85-100 (1972).

16. L. Randeberg, J. Bonesronning, M. Dalaker, J. Nelson, and L. Svaasand, "Methemoglobin formation during laser induced photothermolysis of vascular skin lesions," Lasers in Surgery and Medicine 34(5), 414-419 (2004).

17. M. Meinke, G. Müller, M. Friebel, and J. Helfmann, "Optical properties of platelets and blood plasma and their influence on the optical behavior of whole blood in the visible to near infrared wavelength range," J. Biomed. Opt. 12(1), 014024 (2007).

18. R. M. Pope, and E. S. Fry, Absorption spectrum (380-700 nm) of pure water. II. Integrating cavity measurements, Applied Optics 36(33), 8710-8723 (1997).

19. K. F. Palmer, and D. Williams, "Optical properties of water in the near infrared," Journal of the Optical Society of America 64(8), 1107-1110 (1974).

20. J. Coquin, A. Dewitte, Y. L. Manach, M. Caujolle, O. Joannes-Boyau, C. Fleureau, G. Janvier, and A. Ouattara, "Precision of noninvasive hemoglobin-level measurement by pulse co-oximetry in patients admitted to intensive care units for severe gastrointestinal bleeds," Critical Care Medicine 40(9), 2576-2582 (2012).

21. L. J. Moore, C. E. Wade, L. Vincent, J. Podbielski, E. Camp, D. Junco, H. Radhakrishnan, J. McCarthy, B. Gill, and J. B. Holcomb, "Evaluation of noninvasive hemoglobin measurements in trauma patients," The American Journal of Surgery 206(6), 1041-1047 (2013).

\section{Introduction}

The main advantages of noninvasive methods for the analysis of blood protein components are the removal of the infection risk, the efficiency, the possibility of continuous monitoring, the absence of biologic pollution, and the softened requirements to the personnel qualification. Various methods [1-9] can be used to assess the blood haemoglobin concentration in vivo.

Among these methods, the optical ones are most acceptable for the practical use. This is due to the relative simplicity of the instrumentation required to implement the optical methods of noninvasive haemoglobin concentration and the techniques for clinical applications. The analysis of trends in the development of optical methods and technical means for assessment of haemoglobin concentration shows that the main trend lies in the field of using the method of pulse CO-oximetry $[10,11]$.

The method of pulse CO-oximetry is based on measuring the optical radiation attenuation by the arterial blood at different wavelengths and calculating the concentrations of the total haemoglobin and its fractions from the obtained attenuation values. The number of probe wavelengths determines the number of determined fractions of haemoglobin. Thus, in the commercially produced monitors Rad-57 and Raical-7 (Masimo) twelve wavelengths are used [12, 13] and the concentrations of the total haemoglobin, oxyhaemoglobin, carboxyhaemoglobin, and methaemoglobin are determined. However, in many practical cases, e.g., for the anaemia diagnostics, the information on the fractional composition is abundant, and it is sufficient to know the total haemoglobin concentration solely. In this relation, the question arises about the set of probe radiation wavelengths necessary to determine the blood haemoglobin concentration with acceptable accuracy.

The aim of our study was to estimate the accuracy of measuring the blood haemoglobin concentration by means of the pulse CO-oximetry method depending on the chosen wavelengths of the probe radiation. The determination of the set of wavelengths minimising the measurement error will offer an opportunity to optimise the construction of the instrument for noninvasive assessment of the haemoglobin concentration from the point of view of scheme simplification and, therefore, cost reduction. In turn, the cost reduction and device simplification will allow the implementation of this method in wide clinical practice of the population screening, aimed at revealing the persons with diseases that affect the haemoglobin concentration.

To solve the formulated problem the studies were carried out with the aim of comparing the results obtained using the experimental multiwave system for noninvasive assessment of the blood haemoglobin concentration and the data of clinical blood analysis. The essence of the study was to determine the measurement error for different linear combinations of wavelengths. The experimental examinations were carried out in the group of haematological patients with different pathologies on the base of the Haematology Department of Samara State Medical University. 


\section{Theoretical foundation of the pulse $\mathrm{CO}$ - oximetry}

The method of pulse CO-oximetry is based on the Bouguer-Lambert-Beer law that relates the reduction of the intensity of light that passes through a layer of lightabsorbing matter to the concentration of the absorbing substance and the layer thickness:

$$
I=I_{0} \cdot 10^{-\varepsilon \cdot l \cdot c}
$$

where $\varepsilon$ is the molar absorption coefficient, $l$ is the thickness of light-absorbing layer $(\mathrm{m}), c$ is the concentration of the stained solution (M). For several stained substances present in the solution the contributions are additive, i.e., $A=A_{1}+A_{2}+\ldots+A_{n}$, where $A$ is the total optical density of the solution defined as $-\lg T=A=\varepsilon \cdot l \cdot c$, where $\boldsymbol{T}=\frac{\boldsymbol{I}}{\boldsymbol{I}_{\mathbf{0}}}$.

We assume that the optical path lengths are similar for the optical radiation with difference wavelengths propagating through the sample and that the BouguerLambert-Beer law is valid. Then to determine the concentration of each of $n$ stained substances one has to solve the system of linear equations

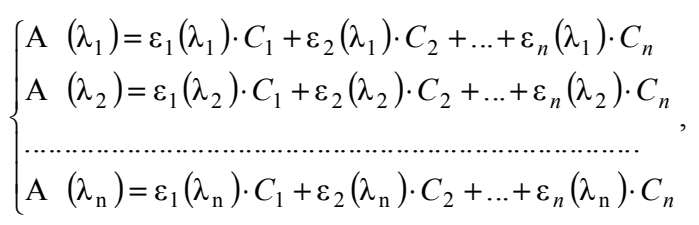

To estimate the amount of haemoglobin $\left(\boldsymbol{C}_{\boldsymbol{H} \text { bTotal }}\right)$, one has to determine the mass of all haemoglobin fractions and the volume of plasma, in which they are dissolved. The direct measurement of these parameters by means of spectrophotometry is impossible, but one can determine the concentration of fractions and then, by plotting the regression dependences found from the blood clinical analysis, estimate the amount of blood haemoglobin.

The main fractions of blood haemoglobin are oxyhaemoglobin $\left(\boldsymbol{C}_{\boldsymbol{H b O}_{2}}\right)$, deoxyhaemoglobin $\left(\boldsymbol{C}_{\boldsymbol{H b}}\right)$, carboxyhaemoglobin $\left(\boldsymbol{C}_{\mathrm{HbCO}_{2}}\right)$, and methaemoglobin $\left(\boldsymbol{C}_{\boldsymbol{H} \boldsymbol{B M e t}}\right)$. Normal blood mainly contains $\boldsymbol{C}_{\mathrm{HbO}_{2}}$ and $\boldsymbol{C}_{\boldsymbol{H} \boldsymbol{b}}$, while the part of dysfunctional fractions $\left(\boldsymbol{C}_{\mathrm{HbCO}_{2}}\right.$, $\boldsymbol{C}_{\boldsymbol{H} \boldsymbol{B M e t}}$ ) does not exceed 3\%. Besides, in the norm the fraction of $\boldsymbol{C}_{\mathrm{HbO}_{2}}$ amounts to nearly $98 \%$. Therefore, the first rough approximation the optical medium can be presented as double-layered, consisting of oxyhaemoglobin and blood plasma. Since the blood plasma consists of $90 \%$ water, the set of equations (2) will take the form

$\left\{\begin{array}{l}\mathrm{A}\left(\lambda_{1}\right)=\varepsilon_{\mathrm{HbO} 2}\left(\lambda_{1}\right) \cdot C_{H b O 2}+\varepsilon_{H 2 O}\left(\lambda_{1}\right) \cdot C_{H 2 O} \\ \mathrm{~A}\left(\lambda_{2}\right)=\varepsilon_{\mathrm{HbO} 2}\left(\lambda_{2}\right) \cdot C_{H b O 2}+\varepsilon_{H 2 O}\left(\lambda_{2}\right) \cdot C_{H 2 O}\end{array}\right.$,

and therefore,

$$
C_{H b O 2}=\frac{\varepsilon_{H 2 O}\left(\lambda_{2}\right) \cdot \mathrm{A}\left(\lambda_{1}\right)-\varepsilon_{H 2 O}\left(\lambda_{1}\right) \cdot \mathrm{A}\left(\lambda_{2}\right)}{\varepsilon_{H b O 2}\left(\lambda_{1}\right) \cdot \varepsilon_{H 2 O}\left(\lambda_{2}\right)-\varepsilon_{H b O 2}\left(\lambda_{2}\right) \cdot \varepsilon_{H 2 O}\left(\lambda_{1}\right)},
$$

$$
C_{H 2 O}=\frac{\varepsilon_{H b O 2}\left(\lambda_{1}\right) \cdot \mathrm{A}\left(\lambda_{2}\right)-\varepsilon_{H b O 2}\left(\lambda_{2}\right) \cdot \mathrm{A}\left(\lambda_{1}\right)}{\varepsilon_{H b O 2}\left(\lambda_{1}\right) \cdot \varepsilon_{H 2 O}\left(\lambda_{2}\right)-\varepsilon_{H b O 2}\left(\lambda_{2}\right) \cdot \varepsilon_{H 2 O}\left(\lambda_{1}\right)} .
$$

The analysis of Eqs. (3) shows that to increase the sensitivity and to reduce the error one has to choose the wavelengths providing a large difference between the absorption coefficients of two different components. The optimal choice of wavelengths is of particular importance when using the pulse CO-oximetry method, in which the measured quantity is the increment of absorption due to the systolic increase of blood volume rather than the absolute absorption. This increment depends upon the haemodynamic specific features of the arterial vessels in the limbs of a particular person, the current functional state, and the conditions of measurement. Usually this increment does not exceed $5-7 \%$, the mean value being $1 \%$, and in the case of haemodynamic pathology it can be as small as a few tenths of per cent.

With the absorption spectra of the main haemoglobin fractions, blood plasma, and water in the visible and near-infrared ranges [14-19] taken into account, the probe wavelengths for the experimental studies were chosen as $520 \mathrm{~nm}, 658 \mathrm{~nm}, 776 \mathrm{~nm}, 839$ $\mathrm{nm}, 974 \mathrm{~nm}, 1348 \mathrm{~nm}$, and $1550 \mathrm{~nm}$. The choice was justified by the fact that at $520 \mathrm{~nm}, 658 \mathrm{~nm}, 776 \mathrm{~nm}$, $839 \mathrm{~nm}$, and $974 \mathrm{~nm}$ the absorption coefficient for different forms of haemoglobin by an order of magnitude or more exceeds that of water. On the contrary, at the wavelengths $1348 \mathrm{~nm}$ and $1550 \mathrm{~nm}$ the absorption by water is essentially greater than the absorption by the haemoglobin fractions.

\section{Experimental system and processing algorithms for noninvasive assessment of haemoglobin concentration}

The system for noninvasive assessment of blood haemoglobin concentration was developed at the Chair of Laser and Biotechnical Systems of Samara State Aerospace University. The system consists of the electronic-optical registration unit and the special software operating under OS Windows. The registration unit incorporates the electronic circuit connected to the optical finger sensor via the optical fibre line. In the registration unit the semiconductor lasers radiating at the wavelengths $520,658,776,839,974,1348$, and $1550 \mathrm{~nm}$ are mounted.

The electric power supply of the laser units and the stabilisation of their temperature are implemented by means of the control circuits that switch the lasers on sequentially and provide the constant pulse power and wavelength of radiation. Through the optical fibre cable, the laser radiation is delivered to the biological object and after passing through it is detected by the broadband InGaAs photodiode. The photocurrent output from the 
photodiode, proportional to the attenuation of the radiation by the biological tissues, arrives at the amplifier and then at the synchronous demodulator, in which the multiplexing and the formation of the signals of the attenuation spectral components occurs. These signals, containing constant and variable components, are digitised by the multichannel delta-sigma ADC and are transmitted via the USB to the personal computer for further processing.

Generally, the algorithms of processing the radiation attenuation signals included the following basic stages: the detection of pulse waves, the estimation of the pulse wave amplitude at each wavelength, the estimation of haemoglobin concentration. The detection of pulse waves was implemented by comparison with the filtered signal threshold. The comparison threshold was chosen as a part of the filtered signal amplitude and was dynamically varied with the variation of the signal depending on the registration conditions. The signal filtering was implemented using a bandpass filter formed by a superposition of high-pass and low-pass filter. The filtering was implemented by averaging the photoplethysmographic signal over all channels for low frequencies and by the signal differentiation for high frequencies. The time constant of the differentiator was chosen to maximise the signal-to-noise ratio.

The signal amplitude was calculated using the maximum likelihood estimate

$$
\hat{A}=\frac{\int_{0}^{T} X(t) S_{1}(t) d t}{\int_{0}^{T} S_{1}^{2}(t) d t},
$$

where $X(t)$ is the current signal in the channel, $S_{I}(t)$ is the reference signal, $[0, \mathrm{~T}]$ is the duration of a single pulse wave. The rectangular signals with different durations and the signal obtained by averaging the pulse wave realisations were used as reference signals. The obtained estimates of amplitudes were analysed for the presence of outliers and filtered using either the sigma clipping algorithm, or the median filter.

After the determination of the sequence of algorithms for the primary processing of signals, optimal from the point of view of the error variance minimisation, the data were used for the estimation of haemoglobin concentration.

To estimate the haemoglobin concentration we used the multifactor linear regression model, implemented using the MATLAB application software. The factors for the regression model were the normalised ratios of radiation attenuations at different wavelengths. These ratios were calculated as

$$
R_{\lambda_{i} / \lambda_{j}}=\frac{\Delta A\left(\lambda_{i}\right) / A\left(\lambda_{i}\right)}{\Delta A\left(\lambda_{j}\right) / A\left(\lambda_{j}\right)},
$$

where $\boldsymbol{A}\left(\boldsymbol{\lambda}_{i, j}\right)$ is the constant component and $\boldsymbol{\Delta} \boldsymbol{A}\left(\boldsymbol{\lambda}_{\boldsymbol{i}, \boldsymbol{j}}\right)$ is the variable component of the attenuation of optical radiation at the $i$-th and $j$-th wavelength, respectively. We could not calculate the factors for the wavelengths 520 and $1550 \mathrm{~nm}$ because of strong attenuation of the signal at these wavelengths and, consequently, the low signal-to-noise ratio. Thus, 8 linearly independent factors were formed, namely $\boldsymbol{R}_{\mathbf{1}}=\boldsymbol{R}_{\lambda_{1} / \lambda_{5}}, \boldsymbol{R}_{\mathbf{2}}=$ $\boldsymbol{R}_{\lambda_{2} / \lambda_{5}}, \boldsymbol{R}_{\mathbf{3}}=\boldsymbol{R}_{\lambda_{3} / \lambda_{5}}, \boldsymbol{R}_{\mathbf{4}}=\boldsymbol{R}_{\lambda_{4} / \lambda_{5}}, \boldsymbol{R}_{\mathbf{5}}=\boldsymbol{R}_{\lambda_{1} / \lambda_{4}}$, $\boldsymbol{R}_{\mathbf{6}}=\boldsymbol{R}_{\lambda_{2} / \lambda_{4}}, \boldsymbol{R}_{\mathbf{7}}=\boldsymbol{R}_{\lambda_{3} / \lambda_{4}}, \quad \boldsymbol{R}_{\mathbf{8}}=\boldsymbol{R}_{\lambda_{2} / \lambda_{3}}$, where $\boldsymbol{\lambda}_{1}=658 \mathrm{~nm}, \boldsymbol{\lambda}_{\mathbf{2}}=776 \mathrm{~nm}, \boldsymbol{\lambda}_{\mathbf{3}}=839 \mathrm{~nm}, \boldsymbol{\lambda}_{\mathbf{4}}=974 \mathrm{~nm}$, and $\lambda_{5}=1348 \mathrm{~nm}$. With these factors the linear model was constructed:

$$
\begin{aligned}
& S H b=a_{0}+a_{1} R_{1}+a_{2} R_{2}+a_{3} R_{3}+a_{4} R_{4}+a_{5} R_{5}+a_{6} R_{6}+ \\
& a_{7} R_{7}+a_{8} R_{8}
\end{aligned}
$$

where $S H b$ is the haemoglobin concentration estimate, $a_{0} \ldots a_{8}$ are the linear model coefficients, determined from the data of clinical analysis.

\section{Results of experimental studies}

The studies were carried out at the Chair of Haematology of Samara State Medical University. The measurements of haemoglobin concentration using the laboratory method ( ) were performed in the group of 90 patients with different pathologies of haematological profile. The range of variation of the haemoglobin concentration in the studied group amounted to 47-190 $\mathrm{g} / \mathrm{L}$, and the haematocrit varied within 15-61\%. Then in the same patients during 30 seconds the multiwave photoplethysmogram was recorded and processed using the above algorithm. The regression model factors were calculated using Eq. (5). Thus, the data matrix was completed in the form

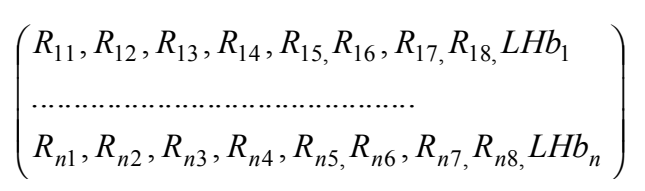

where $n=90$.

Then using 50 randomly chosen lines of the matrix (8) the coefficients for the regression model (7) were calculated and for all patients the values of $S H b$ were determined. The results obtained using the system were compared with the results of the blood clinical analysis. The statistics of the difference $S H b-L H b$ was calculated. In the case of using all 8 factors in the model (6) the root-mean-square deviation $\sigma=20.45 \mathrm{~g} / 1$. the mathematical expectation $\boldsymbol{m}=0$, the median $=-2.3$. The histogram of the differences $S H b-L H b$ for this case is presented in Fig. 1. 


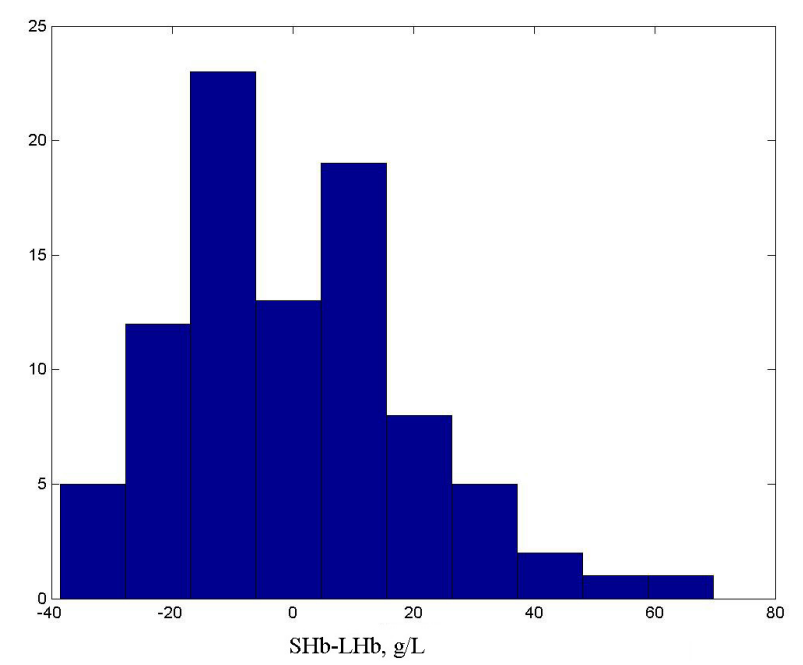

Fig. 1 Histogram for the differences $S H b-L H b$ in the case of using 8 factors.

After that, some factors were excluded from the model (6) to determine such combination of factors, for which the root-mean-square is minimal in the case of using a smaller number of probe wavelengths. It was found that this is achieved with the factors $R_{1}, R_{2}, R_{4}$. In this case the root-mean-square deviation $\boldsymbol{\sigma}=21.0 \mathrm{~g} / 1$, the mathematical expectation $\mathbf{m}=\mathbf{0}$, the median $=$ -1.8 . The histogram of differences $\mathrm{SHb}-\mathrm{LHb}$ for this case is presented in Fig. 2. In this case the mathematical expectation can be considered as the systematic error of the measurement, and the root-mean-square deviation as the random error.

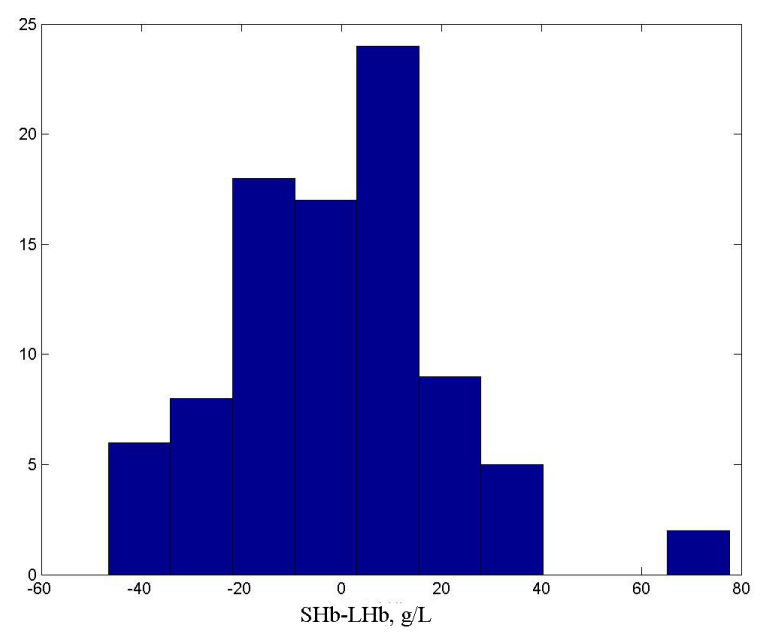

Fig. 2 Histogram for the differences $S H b-L H b$ in the case of using the factors $R_{1}, R_{2}, R_{4}$.

The studies have shown that the exclusion of $\lambda_{3}=839 \mathrm{~nm}$ from the set of probe wavelengths insignificantly increases the root-mean-square deviation, while the mathematical expectation is not changed at all.

The attempt to compare the obtained results with those of the noninvasive haemoglobin measurements carried out using the commercial monitors Raical-7 and
Rad-87 (Masimo) in different clinical investigations shows the following. The data on the measurement accuracy, defined as the root-mean-square deviation $S H b-L H b$ strongly differ in different sources and are hardly comparable. Thus, in the review [10] for different cases the mathematical expectation varies within the range from 0 to $8 \mathrm{~g} / \mathrm{L}$, and the root-meansquare deviation from 5 to $13 \mathrm{~g} / \mathrm{L}$. However, in Ref. [20] $\boldsymbol{m}=10 \mathrm{~g} / \mathrm{l}$, and $\boldsymbol{\sigma}=19 \mathrm{~g} / \mathrm{l}$. The difficulty of comparing these data is related to the difference in ranges of haemoglobin variation in the groups of patients of different age and sex, the lack of data on the quality of the recorded signals of arterial blood pulsation, on whether the outlier rejection was performed or not, the diversity of pathologies, etc.

Another approach to the assessment of noninvasive haemoglobin measurement precision is presented in Ref. [21]. In this paper the authors determine the $95 \%$ confidence interval for the difference $S H b-L H b$. It was found that for the measurement of haemoglobin in the interval from 80 to $170 \mathrm{~g} / \mathrm{L}$ the $95 \%$ confidence interval amounts to $71.8 \mathrm{~g} / \mathrm{L}$.

We carried out the analogous studies and found that in the case of using 8 factors the $95 \%$ confidence interval, obtained by means of our experimental multiwave system appeared to be $73.2 \mathrm{~g} / \mathrm{L}$, and with three factors $R_{1}, R_{2}, R_{4}$ it was $80.7 \mathrm{~g} / \mathrm{L}$, which is worse. However, the range of variation for the haemoglobin concentration in our group was $47-190 \mathrm{~g} / \mathrm{L}$, which is larger than in Ref. [21]. For the range of haemoglobin variation from 80 to $170 \mathrm{~g} / \mathrm{L}$ that corresponds to the measurement range of Masimo monitors, we obtained the $95 \%$ confidence interval $62.3 \mathrm{~g} / \mathrm{L}$ with 8 factors and $65.5 \mathrm{~g} / \mathrm{L}$ with three factors $R_{1}, R_{2}, R_{4}$, using our experimental multiwave system. The root-mean-square deviation amounted to $12.3 \mathrm{~g} / \mathrm{L}$ in the first case and 14.7 $\mathrm{g} / \mathrm{L}$ in the second case. The group with the haemoglobin variation range from 80 to $170 \mathrm{~g} / \mathrm{L}$ was selected from the initial group and included 64 patients.

Since our system of multiwave noninvasive assessment of haemoglobin concentration is aimed at the population screening, the achieved accuracy can be considered acceptable for revealing the persons with potential pathologies. With such measurement error it is possible to reduce the probability of missing the disease by choosing appropriately the diagnostic thresholds, by increasing the measurement time in the case of weak peripheral pulsation signal, and by taking the risk factors and clinical symptoms into account. For example, in the technique for medical application one can set the thresholds of directing the patient to the laboratory examination corresponding to the haemoglobin at the level of the norm limits. However, all this requires further studies aimed at getting the a priori distributions of the haemoglobin concentrations in the population, both for healthy people and those with pathologies affecting the blood haemoglobin concentration. 


\section{Conclusion}

The studies have shown that for assessment of blood haemoglobin concentration with the accuracy comparable with that of commercially produced monitors Rad-57 and Raical-7 (Masimo) it is sufficient to use four wavelengths of probe radiation $\lambda_{1}=658$ $\mathrm{nm}, \lambda_{2}=776 \mathrm{~nm}, \lambda_{4}=974 \mathrm{~nm}$, and $\lambda_{5}=1348 \mathrm{~nm}$. In this case for the haemoglobin concentration range from 80 to $170 \mathrm{~g} / \mathrm{L}$ the root-mean-square deviation that characterises the accuracy and equals to $14.7 \mathrm{~g} / \mathrm{L}$ and the $95 \%$ confidence interval equal to $65.5 \mathrm{~g} / \mathrm{L}$ are none the worse than the values presented in literature. To our opinion, such factor as the quality of the recorded signal affects the accuracy much stronger than the reduction of the probe wavelengths number. Further improvement of the accuracy of the assessment of blood haemoglobin concentration may be achieved by developing highefficiency signal processing algorithms, which could, e.g., adaptively change the measurement time and their own parameters depending on the signal-to-noise ration and the character of noise.

Thus, the exclusion of $\lambda_{3}=839 \mathrm{~nm}$ practically does not worsen the results and allows the simplification of technical implementation, which, in turn, reduces the cost of the system for the noninvasive haemoglobin concentration assessment. 